\title{
Is Early Bilingualism an Advantage for Children's Development Under Age 12?
}

\author{
Jinghan Ruan ${ }^{1, *}$ \\ ${ }^{1}$ BSc Psychology; University of Edinburgh; Edinburgh; United Kingdom; EH8 9AB \\ ${ }^{*}$ Corresponding author. Email: rjh0808@qq.com
}

\begin{abstract}
Recently, learning more than one language becomes more and more prevalent worldwide. Therefore, people questioned whether dual language development is beneficial or not and what is the best age to start learning a second language. Previous studies have found that there is a critical age for language acquisition around age 12. Thus, this paper is going to answer the question that whether early bilingualism is an advantage for children's development under age 12 . In the review of the empirical data from the literature on comparisons between early bilingual and monolingual children's both cognitive and linguistic development. It was found that learn a second language help improve some components of executive control system, whereas there is no significant difference between early bilinguals and monolinguals' language abilities before early adolescence. Generally, the earlier children learn a second language the more exposure and proficiency they will have for both languages. Thus, better cognitive performance they will have later in life. However, those evidence lacks power and controls over confounding variables such as SES and cultural differences, which may be the alternative explanation for those facilitative responses.
\end{abstract}

Keywords: bilingualism, children's development, language acquisition

\section{INTRODUCTION}

As our society has become more international over the past few decades, it is more and more common for young children to be fostered in a bilingual or multiple languages environment. Bilingualism refers to individuals who are able to process two different languages with equal fluency in everyday life. Additionally, it is very prevalent in many parts of the world. For example, the bilingualism rate of the Canadian population raised up from $17.4 \%$ in 2006 to $17.5 \%$ in 2011 [1]. Overall, researches estimated that over $50 \%$ of the world population is bilingualism. Furthermore, previous studies have defined two types of bilingualism depending on the time of second-language acquisition [2]. Firstly, bilinguals who learn to use two languages before school age are classified as early bilinguals. Whereas, late bilinguals are individuals who learn a second language after childhood or during adulthood.

According to the critical period hypothesis $(\mathrm{CPH})$ proposed by Lenneberg in 1967, it was indicated that age is a crucial factor for language acquisition [3]. He suggested that infants' first language acquisition must occur during the critical period which starts at age two and ends around age of 12 or 13 (i.e., puberty). Therefore, it is predicted that children before a critical age could acquire a second language effortless, accurately and fast. Therefore, early bilingualism may have different development with late bilingualism. The debate on advantages and disadvantages of dual language development has been discussed widely already. Furthermore, surprisingly majority of the previous studies have been conducted on young infants. Consequently, this paper is intended to discuss the general influence of early bilingualism on bilinguals' cognitive and linguistic performance before age 12 . And it will discuss both the benefits and drawbacks by illustrating some evidence from a variety of scientific fields including cognitive psychology, developmental psychology, education and linguistics. Answering this question could aid both family and society to foster successful bilingual development and enhance the current education patterns.

\section{CHILDREN'S DEVELOPMENT}

\subsection{Language Development}

Language development is one of the key outcomes, which previous studies on young dual language learners 
(DLLs) have been interested in. The discussion of literatures has focused on the influence of dual language on the following aspects of language abilities: vocabulary development, grammatical development, word comprehension and phonological development. In a longitudinal experiment conducted by De Houwer et al., infants' word comprehension and production were measured by Communicative Development Inventories (CDI) [4]. The participants were 61 young infants recruited from middle-SES families. 30 of them were monolingual Dutch-speaking, and 31 were Dutch-French bilinguals. Reporters were asked to complete both Dutch CDI (N-CDI) and French CDI (F-CDI) when children were 13 and 20 months of age. The findings showed that bilingual infants understood the same number of Dutch words with monolingual children at 13 months. Whereas, there was a significant advantage for bilinguals when comparing their combined word comprehension (Dutch comprehension plus French comprehension) with that of monolingual infants. On average, monolinguals were found to understood $71 \%$ less words than bilingual infants. However, the advantage was reversed at age of 20 months that monolingual children had a better Dutch total comprehension than bilinguals. Additionally, the study had no evidence of consistent differences between groups when comparing the amount of vocabulary.

Nevertheless, another longitudinal study by Vagh and his colleagues had a larger sample size and disagreed with the previous results [5]. In contrast, 85 children from low-income English monolingual and SpanishEnglish bilingual families were recruited at age of 24, 30, or 36 months. It was found that monolinguals had higher vocabularies at age 30 months based on parent reports, and they were also faster in developing vocabulary across toddlerhood than bilingual children. In comparing grammatical development between Spanish-English speaking infants and monolinguals, Gathercole demonstrated that $2^{\text {nd }}$ grade bilingual children perform worse than $5^{\text {th }}$ graders [6,7]. Importantly, it was also indicated that dual language development children were slower in learning "grammatical gender" in Spanish than monolinguals. Instead of focusing on the caparison between monolingual and bilingual infants' linguistic development, more studies have suggested that it is more crucial to consider the effect of other environmental factors. For example, researches identified the amount of language input plays a substantial role in affecting young bilinguals' both vocabulary and grammatical abilities $[8$, $9,10]$.

Moreover, there are some associations between SES levels and bilingual children's dual language development $[7,11]$. In another experiment by Alt et al., they evaluated the cross-linguistic influence in a fastmapping task. Participants were asked to learn novel dinosaurs' novel names, which had either high or low English phonotactic probability [12]. The findings showed that preschool-age and school-age Spanish-
English bilingual children were less accurate in naming tasks than monolinguals with matched SES. Alt et al. also examined bilingual school-age children and monolinguals' performance in learning novel words [13]. However, they were more comprehensive and extended results into phonological knowledge, phonological linking, semantic knowledge. Their results were similar to Alt et al. that there were few differences in novel word learning between $2^{\text {nd }}$-grade bilingual and monolingual children [12].

Overall, studies have demonstrated the considerable variability in language development between young bilinguals and monolinguals. In general, children who have been daily exposed to two languages may have a slight slower and worse in developing most aspects of language than monolingual infants. Predominantly, factors such as amount of exposure to each language, SES level, language usage and the language spoken could influence their performance in distinct languages.

\subsection{Cognitive Development}

Apart from language, cognitive ability is also substantial in children's development and have been investigated by enormous literatures. It was argued that the executive control system may be constantly involved in bilinguals' language production, because of the allocation of attention on target language. Therefore, researchers suggested that the experience of using two languages may be less vulnerable to interference in nonlinguistic tasks and have facilitative effect on the function of executive. Executive function was defined as a collection of high-level cognitive skills in the frontal lobe. Additionally, Miyake et al. proposed that the exclusive control system consists three main components: shifting (cognitive flexibility or task switching), updating information in working memory and inhibition $[14,15]$. Thus, non-verbal tasks such as the Simon or Stroop task have been frequently used to examine early bilinguals' ability of executive function to inhibitition control [16].

In 2005, Bialystok et al. conducted five studies to test the effect of bilingualism on Simon task in three different age groups: young children ( 5 years old), young adults (20 to 30 years old), and older adults (30 to 59 years and 60 to 80 years) [17]. They reported that the positive influence of early bilingualism was present in both young children and elder adults, but there was no difference in performance between bilinguals and monolinguals who were young adults. Nevertheless, this experiment examined bilinguals with distinct histories of immigration, distinct language pairs, and no information of social classes was provided. Therefore, it was questionable that those confounding factors may also affect their performance, which were responsible for the positive results. To address this issue, Bialystok and Viswanathan recruited 90 eight-year-old children who 
were English-speaking monolingual children in Canada, bilinguals who were capable of using English and another language in Canada, and bilinguals in India who spoke English and either Tamil or Telugu [15]. The results showed that bilingual children were more efficient than monolinguals in switching tasks and inhibitory control, but not suppressing responses. More importantly, they found no significant difference in performance between bilingual children in Canada and India. Thus, the influence of different cultures, social classes and immigration histories could all be eliminated. It supported Bialystok et al.'s findings and contributed to a more detailed understanding of the source of that bilingual advantage, that two components of executive control were affected by childhood bilingualism [17].

In another study by Pelham and Abrams, an attentional network task (ANT) was used to examine participants' abilities of executive function. The experiment included English monolinguals, early Spanish-English bilinguals who became fluent in second language before seven years of age, and late SpanishEnglish bilinguals who became fluent in second language no earlier than thirteen-years-old [18]. They reported that executive control benefits were equivalent for both early and late bilingual children. It was also suggested that the cognitive effects associated with bilingualism is not only related to the age of acquiring the second language, but also the duration that children have been fluently bilingual. Various studies have supported the advantage of bilingualism in childhood.

However, it is not without controversy, as there are also some studies disagreed with their opinions. For example, Morton and Harper used Simon task and allocated 34 young bilingual and monolingual children (aged 6-7 years) who had identical ethnic and socioeconomic backgrounds [19]. Researchers reported that both monolingual and bilingual groups were slower and less accurate on incongruent compared with congruent trials, but there were no differences from each other. Moreover, Martin-Rhee and Bialystok demonstrated that cognitive bilingualism advantage only presented in complex tasks requiring control over attention to competing cues [20]. Whereas, bilinguals showed no facilitative performance on tasks that required inhibition of response comparing with monolinguals.

\section{EVIDENCES AND POSSIBLE SOLUTIONS TO ABOVE PROBLEMS}

Over the past few decades, a large number of studies have compared early bilingual learners against some populations of monolinguals and reported different levels of bilingual advantages across different tasks in testing both cognitive and linguistic performance. Even though that majority of them successfully obtained a positive result and thus support their views of bilingual advantages in early childhood. the effect size's reliability and validity were highly skeptical to many researchers because of some issues in experimental designs and replications crisis.

Firstly, the present of confounds during group comparisons in traditional cross-sectional experiment are difficult to control. Thus, more longitudinal researches are needed to avoid undesirable effect of individual differences. However, those studies are very rare, expansive and time-consuming.

Secondly, the sample size of the most literatures is around 50 which is relatively small compared to other psychological researches. Therefore, future studies need to recruit more participants from wider populations in order to increase the generalizability of the early bilingualism effect.

Thirdly, some previously mentioned literatures have been aware of the importance of some factors other than bilingualism itself in affecting children's development. Whereas, many researchers only focused on the comparisons between bilingual and monolingual participants. Environmental confounders which could influence the true effect of early bilingualism such as social class, culture, immigration, previous exposure of each language and language proficiency were completely ignored, thus more considerations and controls are needed to increase the validity $[21,22,23]$.

Another serious problem is the publication bias, which is not only common in studying bilingualism, but also all fields of psychological researches [24]. Paap et al. noted that papers which answering the positive effect of bilingualism in executive functions were contaminated by Type I errors (false positives), and the evidence is consistent with a null effect [25]. Klein proposed that those studies are normally have a small sample size, questionable statistics, and difficulties in replicating their findings [26].

Additionally, the group difference observed in those experiment may have alternative explanations other than bilingualism. Duñabeitia and Carreiras suggested that pre-registration is one of the most efficient ways to help reduce the influence of publication bias on effect-size estimation, which improves the credibility and reproductivity of research findings [27]. Therefore, all good researches could be published without worrying about results' direction of significance.

\section{CONCLUSION}

Since learning a second language becomes more and more common nowadays, educators and especially the parent intent to understand the effect of bilingualism and question whether the age of acquiring will affect it. Based on the evidence being mentioned, young DLLs have similar linguistic abilities such as vocabulary size, with monolinguals when combing their scores of both 
languages. However, early bilinguals do not show considerable better performance than matched monolingual children in language development generally. In contrast, there are much more studies have demonstrated there is a cognitive advantage related to second-language learning on children's development. It was indicated that younger DLLs have better executive functional abilities in switching tasks and inhibitory control than monolinguals.

Nevertheless, those findings were not consistently observed and studies' validity and reliability were skeptical. One explanation is the lack of control over confounding variables such as SES, which could influence participants' performance other than the acquisition of a second language. Furthermore, reproducibility of studies is also very low because of the publication bias. Therefore, experimental designs with more power like longitudinal studies are needed for future studies. Also, more attention should be given to investigate the plausible effect of the confounders, thus more knowledge about bilingualism could be gained. Same with acquisition of the first language, there is also a sensitive period for individual to learn the second one. The environmental and maturational differences between earlier and later DLLs help indicated that acquiring a second language at earlier age is more advantageous. For example, children in critical period could learn another language more quickly and with less effort. Moreover, early acquisition of two languages provides early input from environment and more time for younger bilinguals to practice both languages than late bilinguals. As the early DLLs gain more proficiency of both languages, they will perform better at controlling attention and other cognitive tasks. Overall, the early acquisition of two languages could enhance people's language development, and executive functioning.

\section{AUTHORS' CONTRIBUTIONS} Ruan.

This paper is independently completed by Jinghan

\section{ACKNOWLEDGMENTS}

This paper would not have been completed without the exceptional support of my supervisor, Mina. Her generous assistant, and knowledge have been an inspiration and kept my work on track.

\section{REFERENCES}

[1] Government of Canada, S. C. (2021, June 17). English-French bilingualism reaches new heights. Government of Canada, Statistics Canada. https://www150.statcan.gc.ca/n1/en/catalogue/98200-X2016009.
[2] Mathews, J. (2019, April 25). Perspective | Half of the world is bilingual. What's our problem? The Washington Post. https://www.washingtonpost.com/local/education/ half-the-world-is-bilingual-whats-ourproblem/2019/04/24/1c2b0cc2-6625-11e9-a1b6b29b90efa879_story.html.

[3] Lenneberg, E. H. (1967). The biological foundations of language. Hospital Practice, 2(12), 59-67.

[4] De Houwer, A., Bornstein, M. H., \& Putnick, D. L. (2013). A bilingual-monolingual comparison of young children's vocabulary size: Evidence from comprehension and production. Applied Psycholinguistics, 35(6), 1189-1211. https://doi.org/10.1017/s0142716412000744

[5] Vagh, S. B., Pan, B. A., \& Mancilla-Martinez, J. (2009). Measuring Growth in Bilingual and Monolingual Children's English Productive Vocabulary Development: The Utility of Combining Parent and Teacher Report. Child Development, 80(5), 1545-1563. https://doi.org/10.1111/j.1467-8624.2009.01350.x

[6] Gathercole, V. C. (2002). Chapter 8: Command of the Mass/Count Distinction in Bilingual and Monolingual Children: An English Morphosyntactic Distinction. Language and Literacy in Bilingual Children, 175-206. https://doi.org/10.21832/9781853595721-009

[7] Gathercole, V. C. (2002). Chapter 9: Grammatical Gender in Bilingual and Monolingual Children: A Spanish Morphosyntactic Distinction. Language and Literacy in Bilingual Children, 207-219. https://doi.org/10.21832/9781853595721-010

[8] Gathercole, V. C., \& Thomas, E. M. Ô. N. (2009). Bilingual first-language development: Dominant language takeover, threatened minority language take-up. Bilingualism: Language and Cognition, $12(2)$,

213-237. https://doi.org/10.1017/s1366728909004015

[9] Williams, N., \& Thomas, E. M. Ô. N. (2017). Exploring minority language input sources as means of supporting the early development of second language vocabulary and grammar. Applied Psycholinguistics, 38(4), 855-880. https://doi.org/10.1017/s0142716416000473

[10] Place, S., \& Hoff, E. (2015). Effects and noneffects of input in bilingual environments on dual language skills in $2 \frac{1}{2}$-year-olds. Bilingualism: Language and Cognition, 19(5), 1023-1041. https://doi.org/10.1017/s1366728915000322 
[11] Gathercole, V. C., Thomas, E. M., Roberts, E. J., Hughes, C. O., \& Hughes, E. K. (2013). 2. Why Assessment Needs to Take Exposure into Account: Vocabulary and Grammatical Abilities in Bilingual Children. Issues in the Assessment of Bilinguals, 20-55. https://doi.org/10.21832/9781783090105004

[12] Alt, M., Meyers, C., \& Figueroa, C. (2013). Factors That Influence Fast Mapping in Children Exposed to Spanish and English. Journal of Speech, Language, and Hearing Research, 56(4), 12371248. https://doi.org/10.1044/1092-4388(2012/110092)

[13] Alt, M., Arizmendi, G. D., Gray, S., Hogan, T. P., Green, S., \& Cowan, N. (2019). Novel Word Learning in Children Who Are Bilingual: Comparison to Monolingual Peers. Journal of Speech, Language, and Hearing Research, 62(7), 2332-2360. https://doi.org/10.1044/2019_jslhr-118-0009

[14] Miyake, A., Friedman, N. P., Emerson, M. J., Witzki, A. H., Howerter, A., \& Wager, T. D. (2000). The Unity and Diversity of Executive Functions and Their Contributions to Complex "Frontal Lobe" Tasks: A Latent Variable Analysis. Cognitive Psychology, 41(1), 49-100. https://doi.org/10.1006/cogp.1999.0734

[15] Bialystok, E., \& Viswanathan, M. (2009). Components of executive control with advantages for bilingual children in two cultures. Cognition, 112(3), 494-500. https://doi.org/10.1016/j.cognition.2009.06.014

[16] Stroop, J. R. (1935). Studies of interference in serial verbal reactions. Journal of Experimental Psychology, 18(6), 643-662. https://doi.org/10.1037/h0054651

[17] Bialystok, E., Martin, M. M., \& Viswanathan, M. (2005). Bilingualism across the lifespan: The rise and fall of inhibitory control. International Journal of Bilingualism, 9(1), 103-119. https://doi.org/10.1177/13670069050090010701

[18] Pelham, S. D., \& Abrams, L. (2014). Cognitive advantages and disadvantages in early and late bilinguals. Journal of Experimental Psychology: Learning, Memory, and Cognition, 40(2), 313-325. https://doi.org/10.1037/a0035224

[19] Morton, J. B., \& Harper, S. N. (2007). What did Simon say? Revisiting the bilingual advantage. Developmental Science, 10(6), 719-726. https://doi.org/10.1111/j.1467-7687.2007.00623.x
[20] Martin-Rhee, M. M., \& Bialystok, E. (2008). The development of two types of inhibitory control in monolingual and bilingual children. Bilingualism: Language and Cognition, 11(1), 81-93. https://doi.org/10.1017/s1366728907003227

[21] Carlson, S. M., \& Choi, H. P. (2009, April). Bilingual and bicultural:

Executive function in Korean and American children. Paper presented at the 2009 biennial meeting of the society for research in child development. Denver, Colorado.

[22] Hoff, E., Core, C., Place S., Rumiche, R., SEÑOR, M., \& Parra, M. (2011). Dual language exposure and early bilingual development. Journal of Child Language, $\quad 39(1), \quad 1-27$. https://doi.org/10.1017/s0305000910000759

[23] Place, S., \& Hoff, E. (2011). Properties of Dual Language Exposure That Influence 2-Year-Olds' Bilingual Proficiency. Child Development, 82(6), 1834-1849. https://doi.org/10.1111/j.14678624.2011.01660.x

[24] Nosek, B. A., Cohoon, J., Kidwell, M., \& Spies, J. R. (2016). Estimating the Reproducibility of Psychological Science. https://doi.org/10.31219/osf.io/447b3

[25] Paap, K. R., Johnson, H. A., \& Sawi, O. (2015). Bilingual advantages in executive functioning either do not exist or are restricted to very specific and undetermined circumstances. Cortex, 69, 265278. https://doi.org/10.1016/j.cortex.2015.04.014

[26] Klein, R. M. (2015). On the belief that the cognitive exercise associated with the acquisition of a second language enhances extra-linguistic cognitive functions: Is "Type-I incompetence" at work here? Cortex, 73, 340-341. https://doi.org/10.1016/j.cortex.2015.07.020

[27] Duñabeitia, J. A., \& Carreiras, M. (2015). The bilingual advantage: Acta est fabula? Cortex, 73, 371-372.

https://doi.org/10.1016/j.cortex.2015.06.009 